\title{
Magnetic Control of Electric-Field Domains in Semiconductor Superlattices
}

\author{
T. Schmidt, ${ }^{1,2}$ A. G. M. Jansen, ${ }^{3}$ R. J. Haug, ${ }^{2,4}$ K. v. Klitzing, ${ }^{2}$ and K. Eberl ${ }^{2}$ \\ ${ }^{1}$ IBM Research Division, T. J. Watson Research Center, Yorktown Heights, New York 10598 \\ ${ }^{2}$ Max-Planck-Institut für Festkörperforschung, Heisenbergstrasse 1, 70569 Stuttgart, Germany \\ ${ }^{3}$ Grenoble High Magnetic Field Laboratory, Max-Planck-Institut für Festkörperforschung and CNRS, BP 166X, \\ 38042 Grenoble, France \\ ${ }^{4}$ Institut für Festkörperphysik, Universität Hannover, Appelstrasse 2, 30167 Hannover, Germany
}

(Received 8 April 1998)

Applying magnetic fields up to $B=29 \mathrm{~T}$, we investigate the formation of electric-field domains in Landau-quantized semiconductor superlattices. Surprisingly, the electric-field distribution remains determined by the subband spacing rather than by the cyclotron energy. At the domain boundary, however, strong inter-Landau-level tunneling occurs, which leads to a magnetically tunable number of extra resonances in the current-voltage curve. In addition, we discuss a novel magnetic-field assisted high-field domain also stabilized by inter-Landau-level tunneling. [S0031-9007(98)07497-3]

PACS numbers: 73.20.Dx, 72.20.Ht, 73.40.Gk

Initiated by Esaki and Tsu [1], the physics of semiconductor superlattices (SLs) has evolved into a particularly active field of research. Fundamental phenomena originally predicted for bulk matter could be first observed in SLs, because their lattice period exceeds the atomic lattice constant of the host material by orders of magnitude. Prominent examples are the Bloch oscillations [2,3] and Wannier-Stark localization [4,5].

Another beautiful effect is the formation of electric-field domains. Miniband transport and sequential resonant tunneling lead to a negative differential resistance in semiconductor SLs. If the electron density in such nonlinear systems is large, the uniform electric-field distribution becomes unstable at the onset of the negative differential resistance and two domains of different electric-field strength are formed [6-9]. The formation of electric-field domains results in a series of peaks in the current-voltage curve $I(V)$, which reflect the spacing of the electronic subbands in the SL.

External perturbations can be utilized to introduce additional energy levels and thus influence the formation of electric-field domains in SLs. Recently, for instance, photon-assisted virtual states were observed to strongly modify the electric-field distribution [10]. In this Letter, we study the influence of Landau quantization on the formation of electric-field domains in a GaAs-AlGaAs SL. This case is particularly challenging, since it features an intricate interplay of intra- and inter-Landau-level tunneling and relaxation processes.

The SL was grown by molecular-beam epitaxy on $n^{+}$-type GaAs substrate. It consists of $15 \mathrm{GaAs}$ quantum wells of $9 \mathrm{~nm}$ width, which are separated from each other by $5 \mathrm{~nm}$ thick $\mathrm{Al}_{0.33} \mathrm{Ga}_{0.67} \mathrm{As}$ barriers; i.e., the SL period is given by $d=14 \mathrm{~nm}$. The central $3 \mathrm{~nm}$ of the quantum wells are $n$ doped with Si to $5 \times 10^{17} \mathrm{~cm}^{-3}$. Spacer layers of $5 \mathrm{~nm} \mathrm{GaAs}$ separate the SL from $300 \mathrm{~nm}$ GaAs contact layers, which are $n$ doped to $2 \times 10^{18} \mathrm{~cm}^{-3}$. A transfermatrix calculation predicts the existence of two minibands at energies $E_{1}=36 \mathrm{meV}$ and $E_{2}=141 \mathrm{meV}$. Their spacing is given by $E_{21}=E_{2}-E_{1}=105 \mathrm{meV}$ and their widths are $\Delta E_{1}=2 \mathrm{meV}$ and $\Delta E_{2}=10 \mathrm{meV}$. The doping of the quantum wells provides a nonvanishing electron density in the lower miniband. Neglecting inhomogeneities at the contacts, the electron density inside the quantum wells equals $n_{w}=1.5 \times 10^{11} \mathrm{~cm}^{-2}$, corresponding to a Fermi energy of $E_{F}=5 \mathrm{meV}$.

From this SL, we fabricated mesas with Ohmic contacts and measured their $I(V)$ curves by sweeping the bias voltage from zero to positive values. Figure 1 shows the $I(V)$ curve of a device with $2 \mu \mathrm{m}$ diameter recorded at a temperature of $T=4.2 \mathrm{~K}$. As many as 12 current peaks with strikingly regular voltage spacing are clearly observed. These current peaks reflect the formation of electric-field domains in the SL. In another SL with $5 \times$ lower electron density, we found similar current peaks of reduced magnitude, while an undoped control sample did not show any structure in the $I(V)$ curve as observed previously [8].

The origin of domain formation is a strongly nonlinear dependence of the electron drift velocity $v_{d}$ on the applied electric field $F$, as illustrated in the inset of Fig. 1. This curve exhibits two maxima. The first maximum at $F_{L}$ corresponds to transport in the lowest level $\left(E_{1} \rightarrow E_{1}\right)$, either via miniband conduction or, if scattering processes are very efficient, via sequential resonant tunneling. If the electric field is increased beyond $F_{L}$, the drift velocity decreases due to the detuning of the lowest energy levels of neighboring quantum wells. The drift velocity exhibits a second maximum at $F_{21}$ where the field is strong enough to allow resonant tunneling between $E_{1}$ and $E_{2}$ of neighboring quantum wells $\left(E_{1} \rightarrow E_{2}\right)$.

The formation of electric-field domains is illustrated in the sketch above Fig. 1. At low bias voltages electron transport occurs exclusively in the lowest level, $E_{1} \rightarrow E_{1}$ [Fig. 1(a)]. The electric field is constant throughout the whole structure. If the bias voltage is increased such that 


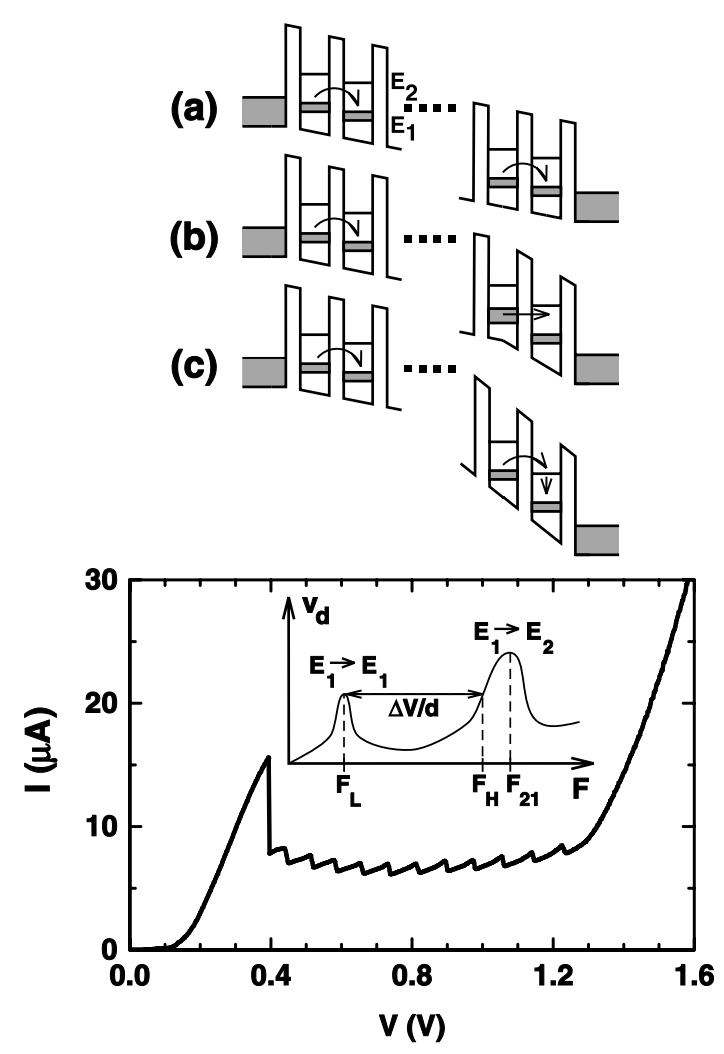

FIG. 1. Current-voltage curve $I(V)$ of the weakly coupled semiconductor superlattice $(T=4.2 \mathrm{~K})$. Multiple current peaks reflect the formation of electric-field domains, as illustrated by sketches (a)-(c). The inset shows the electron drift velocity as a function of electric field.

the average electric field exceeds $F_{L}$, the last quantum well of the SL breaks off from resonant conduction and the current drops abruptly. The resistance increases locally which leads to a higher electron density in the preceding quantum well [Fig. 1(b)]. Thus, the field in the last quantum well exceeds $F_{L}$ and a high-field domain develops. In the rest of the SL forming the low-field domain the electric field remains $F_{L}$. The extra electron density required for the electric-field difference is dynamically accumulated at the domain boundary. Transport occurs via nonresonant tunneling through the corresponding single barrier. By further increasing the bias, the current grows again due to reduction of this single barrier, until $E_{1} \rightarrow E_{2}$ resonant tunneling followed by intersubband relaxation becomes possible [Fig. 1(c)]. By increasing the bias voltage still further, the next quantum well breaks off from resonant conduction, etc., i.e., the high-field domain expands gradually towards the emitter of the SL, while the low-field domain shrinks simultaneously. The number of current peaks corresponds to the number of quantum wells in the SL. We observe slightly fewer current peaks, which can be explained by inhomogeneities at the contacts. At very low or high bias, when one of the two domains is short, several SL periods can switch together [11].

The current peaks should occur at bias voltages of $V_{k}=$ const $+k E_{21} / e$, such that consecutive cur- rent peaks are separated by the subband spacing, $\Delta V=E_{21} / e=105 \mathrm{mV}$ ( $k$ numbers the quantum wells of the SL). This value would be correct if the field were zero in the low-field domain and $E_{21} / e d$ in the high-field domain. Instead, $F_{L}>0$ and $F_{H}<E_{21} / e d$ hold since the drift velocities in the low- and high-field domain have to be identical to allow current conservation throughout the SL [11] (see the inset of Fig. 1). Hence, we measure a smaller peak spacing of $\Delta V=77 \pm 9 \mathrm{mV}<E_{21} / e$. To compare $\Delta V$ and $E_{21} / e$, we have to take scattering into account. Both levels $E_{1}$ and $E_{2}$ exhibit a scatteringinduced broadening $\hbar / \tau_{1}$ and $\hbar / \tau_{2}$ due to interface roughness and impurity scattering [12]. For resonant tunneling in the high-field domain, it is sufficient if the top edge of the broadened level $E_{1}$ is aligned with the bottom edge of the broadened level $E_{2}$ in the following quantum well, i.e., the peak spacing is given by

$$
\Delta V \sim E_{21} / e-\eta\left(\hbar / \tau_{1}+\hbar / \tau_{2}\right),
$$

where $\eta$ is a factor of order unity [7]. The broadening of $E_{1}$ can be estimated from the low-bias part of the $I(V)$ curve. Transport through $E_{1}$ works as long as the voltage drop across one SL period does not exceed the scattering-induced broadening, i.e., until the bottom edge of $E_{1}$ crosses the top edge of $E_{1}$ in the following quantum well. In our samples, transport through $E_{1}$ works up to $V_{1} \sim 300-400 \mathrm{mV}$, which corresponds to a broadening of $\hbar / \tau_{1}=e V_{1} / 15 \sim 20-25 \mathrm{meV}$. Assuming $\hbar / \tau_{2}=\hbar / \tau_{1}$, we obtain $\eta=0.6-0.7$ from Eq. (1). The scattering-induced broadening exceeds the miniband width $\Delta E_{1}=2 \mathrm{meV}$ by 1 order of magnitude. Therefore, sequential resonant tunneling dominates over miniband conduction at low bias voltages.

Now, we investigate the formation of electric-field domains at high magnetic fields applied parallel to the direction of current flow. Figure 2 shows the $I(V)$ characteristics for $0 \leq B \leq 29 \mathrm{~T}$. The most striking feature of the data is a multiplication of the number of current peaks at intermediate magnetic fields. At ultrahigh fields, the $I(V)$ curves are again similar to that at $B=0 \mathrm{~T}$, except for the reduction of the very first current peak. For a detailed analysis of the data, we plot in Fig. 3(a) the numerically obtained differential conductance $G=d I / d V$ as a function of bias voltage and magnetic field. White lines reflect regions of strongly negative differential conductance, i.e., they indicate the positions of the sharp current drops that follow the peaks in the $I(V)$ data. In the regime of intermediate fields of $5<B<20 \mathrm{~T}$, two additional resonances appear between each pair of the original resonances present at $B=0 \mathrm{~T}$. These fan-type extra resonances move with increasing magnetic field to higher bias voltage [13].

To reveal the origin of the extra resonances, we consider the formation of Landau levels (LLs) $E_{i, n}=$ $E_{i}+(n+1 / 2) \hbar \omega_{c}$ in the SL, where $i=1,2$ is the subband index, $n=0,1, \ldots$ is the Landau quantum number, and $\omega_{c}=e B / m^{*}$ is the cyclotron frequency. 
(a)
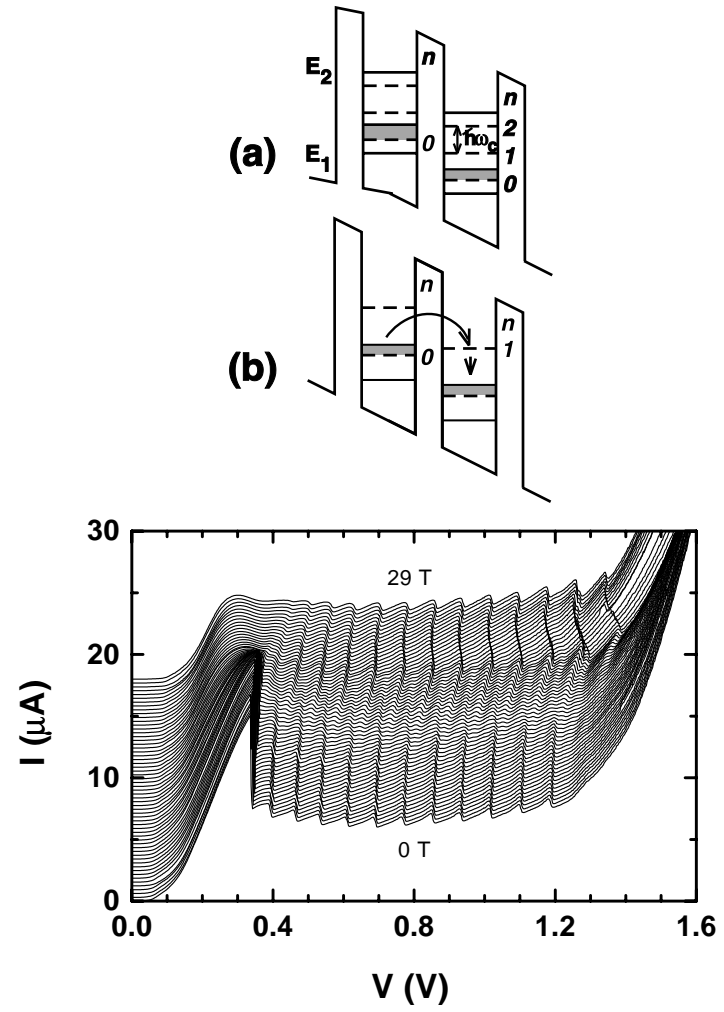

FIG. 2. Magnetic-field dependence of the $I(V)$ curve $(T=$ $0.5 \mathrm{~K}$ ). The curves are plotted with a vertical offset (step $0.5 \mathrm{~T}$ ). Sketch (a) illustrates inter-LL tunneling at the domain boundary. Sketch (b) shows the formation of a magnetic-field assisted high-field domain.

According to the equilibrium electron density of $n_{w}=1.5 \times 10^{11} \mathrm{~cm}^{-2}$, the SL is at $B>3 \mathrm{~T}$ in the magnetic quantum limit. In a first step, we restrict ourselves to this case, in which only the lowest (spindegenerate) LL is occupied.

In the absence of scattering, tunneling conserves the Landau index. Inter-LL tunneling is forbidden, since the in-plane wave functions of the LLs are orthogonal harmonic oscillator eigenfunctions. Under these conditions, $E_{1,0} \rightarrow E_{1,0}$ and $E_{1,0} \rightarrow E_{2,0}$ transport replaces the $E_{1} \rightarrow E_{1}$ and $E_{1} \rightarrow E_{2}$ processes in the low- and highfield domains. In contrast to our data, no extra peaks would show up in the $I(V)$ curve.

Elastic scattering processes such as interface roughness or impurity scattering as well as optical-phonon emission lead to inter-LL tunneling, which was observed both in double-barrier heterostructures $[14,15]$ and in SLs [16,17]. In the case of strong elastic scattering, as expected for our doped SL, resonant inter-LL tunneling $E_{1,0} \rightarrow E_{1, m}$ at the domain boundary leads to extra resonances between each pair of original resonances at bias voltages of

$$
\Delta V_{m}=m\left(\hbar \omega_{c}\right) / e \alpha<\Delta V,
$$

as illustrated in sketch (a) of Fig. 2. In Eq. (2), $\alpha$ denotes the voltage-to-energy conversion coefficient involved. In agreement with our data, these extra resonances move with increasing magnetic field to higher bias voltage.
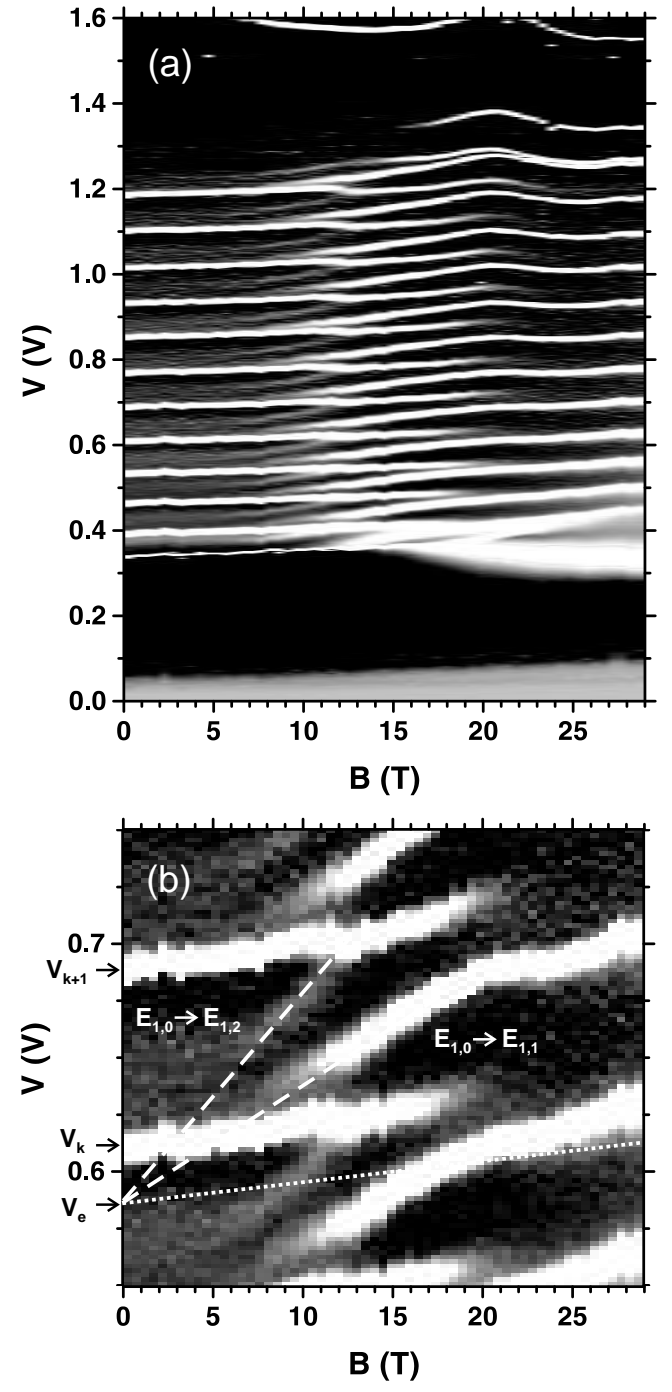

FIG. 3. (a) Grey-scale plot of the differential conductance $G=d I / d V$ versus bias voltage and magnetic field (white: $G \leq-7 \mu \mathrm{S}$; black: $G \geq 15 \mu \mathrm{S}$ ). (b) Detail of plot (a). Dashed lines extend the extra resonances to $B=0 \mathrm{~T}$. The dotted line runs in parallel to the main resonances starting at the point of extrapolation.

Experimentally, the extra resonances are less pronounced in the SL with $5 \times$ lower doping concentration, which supports the conclusion that their formation involves impurity scattering.

For a quantitative comparison, we consider Fig. 3(b). The dashed white lines extend the extra resonances to zero magnetic field. They do not extrapolate to the bias voltage of the original resonance at $V_{k}=0.61 \mathrm{~V}$ but to a lower absolute value $V_{e}$. This difference reflects the broadening of the LLs, since the spacing of $V_{e}$ and the next original resonance $V_{k+1}$ coincides with $E_{21} / e$. Thus, we ascribe the extra resonances to $E_{1,0} \rightarrow E_{1,1}$ and $E_{1,0} \rightarrow$ $E_{1,2}$ tunneling. Using the magnetic-field dependence of the main resonances as a reference (dotted line), we obtain $\alpha=0.4$ from Eq. (2). This value is close to the voltage-to-energy conversion coefficient in double-barrier 
heterostructures, which is not surprising since the domain boundary extends over one SL period [8].

The magnetic-field independence of the main-resonance spacing $\Delta V$ shows that, despite the possibility of elastic scattering, transport in the low- and high-field domains occurs via $E_{1,0} \rightarrow E_{1,0}$ and $E_{1,0} \rightarrow E_{2,0}$ intra-LL tunneling. According to Eq. (2), the extra resonances should intersect the next main resonance, when $\Delta V_{m}$ reaches $\Delta V$ or, equivalently, when $m\left(\hbar \omega_{c}\right) / \alpha=E_{21}$, corresponding to $B=12$ and $25 \mathrm{~T}$ for $E_{1,0} \rightarrow E_{1,2}$ and $E_{1,0} \rightarrow E_{1,1}$. Experimentally, the $E_{1,0} \rightarrow E_{1,2}$ resonances vanish at $12 \mathrm{~T}$, while the $E_{1,0} \rightarrow E_{1,1}$ resonances turn into the main resonance close to $20 \mathrm{~T}$. The extra resonances disappear rather than intersecting the main resonances, because the quantum well at the domain boundary joins the highfield domain such that efficient intra-LL transport replaces inter-LL tunneling [18].

The domain boundary reaches the magnetic quantum limit at a higher magnetic field than the rest of the SL. From the electric-field difference between the domains, we estimate an accumulated electron density of $\Delta n_{w} \sim \Delta V / \epsilon \epsilon_{0} d=3.8 \times 10^{11} \mathrm{~cm}^{-2}$. Thus, the quantum well at the domain boundary reaches the quantum limit at $B \sim 11 \mathrm{~T}$. At lower fields, this quantum well contains several occupied LLs and $E_{1, n} \rightarrow E_{1, n+m}$ interLL tunneling across the domain boundary has to be taken into account. However, such additional processes do not lead to new resonances, since Eq. (2) is valid independent of $n$ : for instance, $E_{1,1} \rightarrow E_{1,3}$ tunneling simply intensifies the $E_{1,0} \rightarrow E_{1,2}$ resonances.

Interestingly, our model is independent of the electron density in the SL as long as $E_{F} \ll E_{21}$. If a SL is not in the magnetic quantum limit, $E_{1, n} \rightarrow E_{1, n}$ and $E_{1, n} \rightarrow E_{2, n}$ intra-LL tunneling will occur in the domains without generating new extra resonances.

A more quantitative, theoretical analysis of the interplay of domain formation and Landau quantization is very difficult. Recently, a first effort in this direction has been undertaken [19]. However, it was not possible to take due account for inter-LL tunneling in the SL.

Returning to Fig. 3, we note that the main resonances move to higher bias with increasing magnetic field. The Fermi energy in the emitter contact remains essentially constant but $E_{1,0}$ moves to higher energy; i.e., the bias voltage has to be increased to sustain the electron flow from the emitter into the SL. At $B>15 \mathrm{~T}$, the main resonances exhibit oscillatory features (predominantly at high bias when the domain boundary approaches the emitter), which gives evidence of Landau-band depopulation in the contacts [20].

Finally, we discuss a novel magnetic-field assisted highfield domain. Provided that inter-LL tunneling is sufficiently strong, a high-field domain can be formed in which the current is carried by $E_{1,0} \rightarrow E_{1,1}$ transport [sketch (b) of Fig. 2]. Magnetic-field dependent resonances at $V_{k} \sim$ const $+k\left(\hbar \omega_{c}\right) / e$ should evolve from the very first current peak in the $I(V)$ curve. As a consequence of scattering-induced broadening, these resonances can be observed only if $\Delta V^{\prime} \sim \hbar \omega_{c} / e-\eta\left(\hbar / \tau_{1}+\hbar / \tau_{2}\right)>0$, corresponding to $B>16 \mathrm{~T}$ [Eq. (1)]. Figure 2 shows that the shape of the first current peak changes beyond $15 \mathrm{~T}$. Accompanied by a significant reduction in current, its width starts to increase with magnetic field. This shape change is possibly a precursor of the magnetic-field assisted high-field domain.

In conclusion, the formation of electric-field domains in Landau-quantized semiconductor superlattices is governed by the subband spacing rather than by the cyclotron energy. At intermediate magnetic fields, fan-type series of magnetically tunable extra resonances occur in the $I(V)$ curve due to scattering-assisted inter-LL tunneling at the domain boundary. Within the domains, intra-LL tunneling dominates over a wide range of bias voltages independent of magnetic field.

One of us (T.S.) thanks the Grenoble High Magnetic Field Laboratory for hospitality and the Alexander von Humboldt Foundation for support. We also thank G. Platero and E. E. Mendez for stimulating discussions.

[1] L. Esaki and R. Tsu, IBM J. Res. Dev. 14, 61 (1970).

[2] J. Feldmann et al., Phys. Rev. B 46, 7252 (1992).

[3] C. Waschke et al., Phys. Rev. Lett. 70, 3319 (1993).

[4] E.E. Mendez, F. Agullo-Rueda, and J. M. Hong, Phys. Rev. Lett. 60, 2426 (1988); P. Voisin et al., ibid. 61, 1639 (1988).

[5] K. H. Schmidt et al., Phys. Rev. Lett. 72, 2769 (1994).

[6] L. Esaki and L. L. Chang, Phys. Rev. Lett. 33, 495 (1974).

[7] K. K. Choi et al., Phys. Rev. B 35, 4172 (1987).

[8] H. T. Grahn et al., Phys. Rev. Lett. 67, 1618 (1991).

[9] F. Prengel, A. Wacker, and E. Schöll, Phys. Rev. B 50, 1705 (1994); R. Aguado et al., Phys. Rev. B 55, 16053 (1997), and references therein.

[10] B. J. Keay et al., Phys. Rev. Lett. 75, 4098 (1995).

[11] See, e.g., Semiconductor Superlattices, edited by H.T. Grahn (World Scientific, Singapore, 1995).

[12] The quantum-confined Stark shift of $E_{1}$ and $E_{2}$ is negligible compared with the scattering-induced broadening.

[13] The noise level in Fig. 3 increases towards higher bias reflecting increasing instabilities [for multistability and current oscillations in SLs, see Y. Zhang et al., Phys. Rev. Lett. 77, 3001 (1996), and references therein].

[14] M. L. Leadbeater et al., Phys. Rev. B 39, 3438 (1989).

[15] G. S. Boebinger et al., Phys. Rev. Lett. 65, 235 (1990); Phys. Rev. B 47, 16608 (1993).

[16] W. Müller et al., Phys. Rev. B 46, 9800 (1992).

[17] L. Canali et al., Phys. Rev. Lett. 76, 3618 (1996).

[18] At similar magnetic fields, electron-optical-phonon coupling becomes important. Magnetopolaron formation makes the extra resonances magnetic-field independent at $m\left(\hbar \omega_{c}\right) \sim \hbar \omega_{L O}$ [16], corresponding to $B=10$ and $21 \mathrm{~T}$ for $E_{1,0} \rightarrow E_{1,2}$ and $E_{1,0} \rightarrow E_{1,1}\left(\hbar \omega_{L O} \approx 36 \mathrm{meV}\right)$.

[19] R. Aguado and G. Platero, cond-mat/9803039.

[20] See, e.g., T. Schmidt et al., Phys. Rev. Lett. 78, 1540 (1997), for a discussion of Landau-band depopulation in the emitter and resonant transport. 